\title{
3 A UTILIZAÇÃO DAS FONTES DE INFORMAÇÃO EM SAÚDE COMO PREDITOR DA LITERACIA EM SAÚDE SOBRE USO DE SUBSTÂNCIAS NOS ADOLESCENTES
}

\author{
Catarina Tomás ${ }^{1}$; Paulo Queirós ${ }^{2}$; Teresa Ferreira ${ }^{3} \mid$
}

\section{RESUMO}

CONTEXTO: É a necessidade ou falta de conhecimento que leva à procura de informação, sendo essencial compreender os contextos desta procura com vista a uma estratégia de abordagem da literacia em saúde.

OBJETIVO(S): Conhecer as fontes de informação em saúde utilizadas por uma amostra de adolescentes portugueses e os seus níveis de literacia sobre uso de substâncias, bem como conhecer o impacto da utilização dessas fontes de informação na literacia em saúde estudada. METODOLOGIA: O estudo realizado é quantitativo, descritivo-correlacional, transversal, sendo a amostra de conveniência não probabilística de 1215 adolescentes (idades entre os 14 e os 20 anos, ambos os sexos e a frequentar o ensino secundário no distrito de Leiria). $\mathrm{O}$ questionário de autopreenchimento continha questões sociodemográficas, a Health Literacy Questionnaire for Children e o Instrumento de Avaliação da Literacia em Saúde.

RESULTADOS: A amostra apresenta bons níveis de literacia em saúde em uso de substâncias, utilizando com mais frequência as fontes de informação em saúde o médico e a família. Percebeu-se uma correlação entre os dois conceitos, com uma predição da literacia em saúde em uso de substâncias pela utilização das fontes de informação.

CONCLUSÃO: Existe uma relação explicativa entre os dois conceitos estudados, sendo os níveis de literacia em saúde em uso de substâncias encontrados na amostra bons, e as fontes de informação em saúde utilizadas de forma frequente.

PALAVRAS-CHAVE: Adolescente; Comportamento de procura de droga; Alfabetização em saúde; Comportamento de busca de informação

\section{RESUMEN}

"El uso de las fuentes de información en salud como predictor de la alfabetización en salud en el consumo de sustancias en adolescentes"

CONTEXTO: Es la necesidad o la falta de conocimiento que lleva a la búsqueda de información, y es esencial entender el contexto de esta demanda con el fin de una estrategia de enfoque de la alfabetización en salud.

OBJETIVO(S): Conocer las fuentes de información de salud que utiliza una muestra de adolescentes portugueses y sus niveles de alfabetización en el consumo de sustancias, así como conocer el impacto de la utilización de estas fuentes de información sobre la alfabetización de la salud.

METODOLOGÍA: El estudio es cuantitativo, descriptivo y correlacional, transversal, y la muestra es de conveniencia no probabilística de 1215 adolescentes (de 14 a 20 años, de ambos los sexos y de la escuela secundaria en el distrito de Leiria). El cuestionario auto administrado contenía preguntas sociodemográficas, el Health Literacy Questionnaire for Children y el Instrumento de Avaliação da Literacia em Saúde.

RESULTADOS: La muestra cuenta con buenos niveles de alfabetización en salud en el uso de sustancias, utilizando con mayor frecuencia las fuentes de información de salud médico y familia. Se observó una correlación entre los dos conceptos, con una predicción de la alfabetización en salud en el uso de sustancias por el uso de fuentes de las información.

CONCLUSIÓN: Existe una relación explicativa entre los dos conceptos estudiados, los niveles de alfabetización en salud en el uso de sustancias que se encuentran en la muestra son buenos, y las fuentes de información de salud utilizadas con frecuencia.

\section{DESCRIPTORES: Adolescente; Comportamiento de búsqueda de drogas; Alfabetización en Salud; Conducta en la búsqueda de Información}

\section{ABSTRACT}

"The use of health information sources as a predictor of health literacy in substance use in adolescents"

BACKGROUND: It is the need or lack of knowledge that leads to the search for information, being essential to understand the context of this demand with a view to health literacy approach strategy.

AIM: To know the health information sources used by a sample of Portuguese adolescents and their literacy levels in substance use, as well as knowing the impact of using these sources of information on health literacy.

METHODS: The study is quantitative, descriptive and correlational, cross, and the sample is non-probabilistic by convenience of 1215 adolescents (aged 14 to 20 years, both sexes and in secondary school in the district of Leiria). The self-administered questionnaire contained sociodemographic questions, the Health Literacy Questionnaire for Children and the Instrumento de Avaliação da Literacia em Saúde.

RESULTS: The sample has good health literacy levels in substance use, using more often the health information sources doctor and family. It was noticed a correlation between the two concepts, with a health literacy in substance use prediction by the use of information sources.

CONCLUSION: There is an explanatory relationship between the two studied concepts, the health literacy levels in the use of substances found in the sample are good, and health information sources are frequently used.

KEYWORDS: Adolescent; Drug-seeking behavior; Health literacy; Information seeking behavior

Submetido em 28-10-2015

Aceite em 10-02-2016

1 Doutorada em Ciências de Enfermagem; Enfermeira Especialista em Enfermagem de Saúde Mental e Psiquiatria; Assistente de 2 Triénio no Departamento de Ciências de Enfermagem da Escola Superior de Saúde do Instituto Politécnico de Leiria e Instituição, Morro do Lena - Alto do Vieiro, Leiria, Portugal, catarina.tomas@ipleiria.pt

2 Pós-Doutorado em Ciências de Enfermagem; Enfermeiro Especialista em Reabilitação; Professor Coordenador em Escola Superior de Enfermagem de Coimbra, 3046-851 Coimbra, Portugal, pauloqueiros@esenfc.pt

3 Doutorada em Psicologia; Enfermeira Especialista em Enfermagem de Saúde Mental e Psiquiatria; Professora Coordenadora em ESENF, Portugal, teresarodrigues@esenf.pt

Citação: Tomás, C., Queirós, P. \& Ferreira, T. (2016). A utilização das fontes de informação em saúde como preditor da literacia em saúde sobre uso de substâncias nos adolescentes. Revista Portuguesa de Enfermagem de Saúde Mental (Spe. 3), 15-20. 


\section{INTRODUÇÃO}

A procura de informação ocorre sobretudo em resposta a uma necessidade ou falta de conhecimento (Mukherjee \& Bawden, 2012), devendo olhar-se para as fontes de informação como parte da estratégia para abordar a literacia em saúde (Norman \& Skinner, 2006). Fatores como a necessidade de conhecimento, bem como o ambiente e contexto onde a informação é necessária influenciam a forma como esta mesma informação é recolhida, nomeadamente as fontes privilegiadas e a frequência da sua utilização (Mukherjee \& Bawden, 2012).

Vários estudos foram já realizados no sentido de compreender quais as fontes de informação em saúde mais valorizadas pelos adolescentes, bem como a sua frequência de utilização. Fontes como os pais, a internet, os amigos e os profissionais de saúde surgem como as mais utilizadas por esta faixa etária, seguidas das revistas, televisão, professores e panfletos. Os profissionais de saúde, os professores, os panfletos e os pais surgem como as fontes com maior credibilidade percebida pelos adolescentes (Dobransky \& Hargittai, 2012, Kwan, Arbour-Nicitopoulos, Lowe, Taman, \& Faulkner, 2010, Nustad, Adams, \& Moore, 2008). As raparigas procuram mais informação sobre saúde, sobretudo nas fontes de informação panfletos, amigos e revistas (Kwan et al., 2010).

Fontes eletrónicas como a internet fazem cada vez mais parte dos contextos de informação em saúde, sendo bastante utlizados pela população em geral, e em particular pelos adolescentes (Andreassen, et al., 2007, Austin, 2012; Ghaddar, Valerio, Garcia, \& Hansen, 2012; Gray, Klein, Noyce, Sesselberg, \& Cantrill, 2005; Norman \& Skinner, 2006a; Stellefson et al., 2011). Nenhum dos sexos parece demonstrar dúvidas ou preocupações referentes à veracidade e fidedignidade da informação obtida, sendo percetível que os rapazes procuram mais informação de serviços e produtos para adquirir, enquanto as raparigas procuram mais informação sobre diagnósticos (Stellefson et al., 2011).

Relacionando os níveis de literacia com a utilização das fontes de informação, existem estudos que demonstram que a utilização de fontes como os jornais, revistas, televisão e panfletos é maior em pessoas com níveis de literacia baixos, enquanto fontes como a internet, revistas, panfletos e jornais são utilizadas por pessoas com níveis mais elevados de literacia em saúde.
Também a frequência de utilização destas fontes é superior nos indivíduos com níveis mais elevados de literacia em saúde, bem como a utilização mais prevalente de fontes como os profissionais de saúde, amigos e familiares (Kutner, Greenberg, Jin, Paulsen, \& White, 2006). Pesquisar e utilizar mais as fontes de informação em saúde relaciona-se assim com níveis mais elevados de literacia em saúde (Paek, Reber, \& Lariscy, 2011).

O consumo de substâncias surge de forma recorrente como um dos tópicos acerca dos quais os jovens referem sentir necessidade e interesse em encontrar informação (Gray, Klein, Noyce, Sesselberg, \& Cantrill, 2005), sendo também um dos tópicos de saúde sobre o qual referem receber mais informação. Os rapazes afirmam receber mais informação acerca deste tema do que as raparigas (Ackard \& Neumark-Sztainer, 2001, Al-Qallaf, Al-Otaibi, \& Othman, 2012 e Kwan et al., 2010).

Apesar da informação recebida acerca dos vários tópicos em saúde, os níveis baixos de literacia em saúde nos adolescentes rondam os 10 a 40\% (Sanders, Federico, Klass, Abrams, \& Dreyer, 2009), sendo os níveis de literacia em saúde superiores no sexo feminino, e nos jovens com nacionalidade, naturalidade ou língua estrangeira (Wu, et al., 2010).

\section{METODOLOGIA}

Pretendeu-se com este estudo responder a dois objetivos. O primeiro refere-se a conhecer as fontes de informação em saúde utilizadas por uma amostra de adolescentes portugueses, bem como os seus níveis de literacia em saúde sobre uso de substâncias. O segundo objetivo é o de conhecer o impacto da utilização das fontes de informação em saúde na literacia em saúde sobre uso de substâncias, nessa mesma amostra.

O estudo que se apresenta é quantitativo, descritivocorrelacional e transversal, utilizando um amostra de conveniência de 1215 adolescentes, com idade entre os 14 e os 20 anos, de ambos os sexos, a frequentar o ensino secundário no distrito de Leiria, selecionada por um método de amostragem não probabilístico. Foram definidos como critérios de inclusão a aceitação de participação no estudo, a idade dentro dos limites definidos e a frequência de ensino secundário no distrito de Leiria. Os critérios de exclusão aplicados foram a não frequência das escolas selecionadas para constituição da amostra e a não autorização para participação por parte do encarregado de educação no caso de jovens menores. 
A colheita de dados, realizada em 2013, foi realizada utilizando um questionário de autopreenchimento composto por três partes. A primeira parte consistia em questões de caracterização sociodemográfica. A segunda parte era constituída pelo Health Literacy Questionnaire for Children (Vardavas, Kondilis, Patelarou, Akrivos, \& Falagas, 2009, traduzido e validado para a população portuguesa por Tomás, 2014) e a terceira parte pelo Instrumento de Avaliação da Literacia em Saúde elaborado por Tomás (2014). A utilização das fontes de informação em saúde foi avaliada através do instrumento Health Literacy Questionnaire for Children. Trata-se de um instrumento que avalia vários componentes da literacia em saúde, nomeadamente os tópicos de saúde sobre os quais os jovens receberam informação no último ano, as fontes de informação em saúde utilizadas e a satisfação em relação à interação com o profissional de saúde. A utilização das fontes de informação em saúde é avaliada partindo de oito questões de resposta tipo Likert com cinco opções de resposta (de Nunca a Sempre) e pontuação de 1 a 5, sendo que valores mais elevados correspondem a uma frequência de utilização da fonte de informação mais elevada.

A literacia em saúde sobre uso de substâncias foi avaliada utilizando o Instrumento de Avaliação da Literacia em Saúde, um instrumento que avalia a literacia em saúde em 9 temáticas diversas e 7 parâmetros de desempenho em literacia. É um instrumento composto por 11 cenários e 52 questões de escolha múltipla (resposta dicotómica), tendo sido considerado 1 cenário e 5 questões para avaliação da literacia em saúde sobre uso de substância. Havendo a possibilidade de pontuar entre 0 e 1 neste instrumento, valores mais elevados de literacia correspondem a pontuações mais elevadas.

Todos os procedimentos formais e éticos inerentes a este tipo de estudo foram tido em conta, tendo os dados sido tratados com recurso a testes paramétricos.

\section{RESULTADOS}

A amostra do estudo foi constituída por adolescentes de idades entre os 14 e os 20 anos, sendo a média de idade de 16,31 anos e a moda de 16 anos. Ambos os sexos se encontravam representados, sendo $52,76 \%$ dos adolescentes do sexo masculino e $47,24 \%$ do sexo feminino. Distribuídos pelos três anos de escolaridade do ensino secundário, a amostra apresentava elementos dos quatro cursos científico-humanísticos, bem como dos cursos profissionais lecionados nas instituições de ensino selecionadas para constituição da mesma.
A maioria dos jovens frequentava o $11^{\circ}$ ano de escolaridade $(38,68 \%)$ e o curso científico-humanístico de ciências e tecnologias (52,43\%). Em termos culturais, a grande maioria dos participantes referiram ser de nacionalidade $(96,13 \%)$ e naturalidade portuguesa $(93,99 \%)$, dominando a língua portuguesa para comunicar com a família $(97,45 \%)$, amigos e nas relações sociais $(97,45 \%)$ e para ler e escrever $(97,78 \%)$.

Encontraram-se níveis satisfatórios de literacia em saúde (Tabela 1) sobre uso de substâncias, sendo este valor mais elevado nos adolescentes do sexo feminino em detrimento do sexo masculino. Os adolescentes a frequentar o $12^{\circ}$ ano de escolaridade apresentam também os valores mais elevados e os adolescentes a frequentar o $10^{\circ}$ ano de escolaridade os valores mais baixos. Não se encontraram relações entre os níveis de literacia em saúde sobre uso de substâncias e a idade dos jovens, quer comparando grupos etários, quer em termos correlacionais $(\mathrm{r}=-0,012 ; \mathrm{p}=0,674)$

Tabela 1 - Níveis de literacia em saúde sobre uso de substâncias (Teste t-student, One-Way ANOVA e Post-Hoc Tukey)

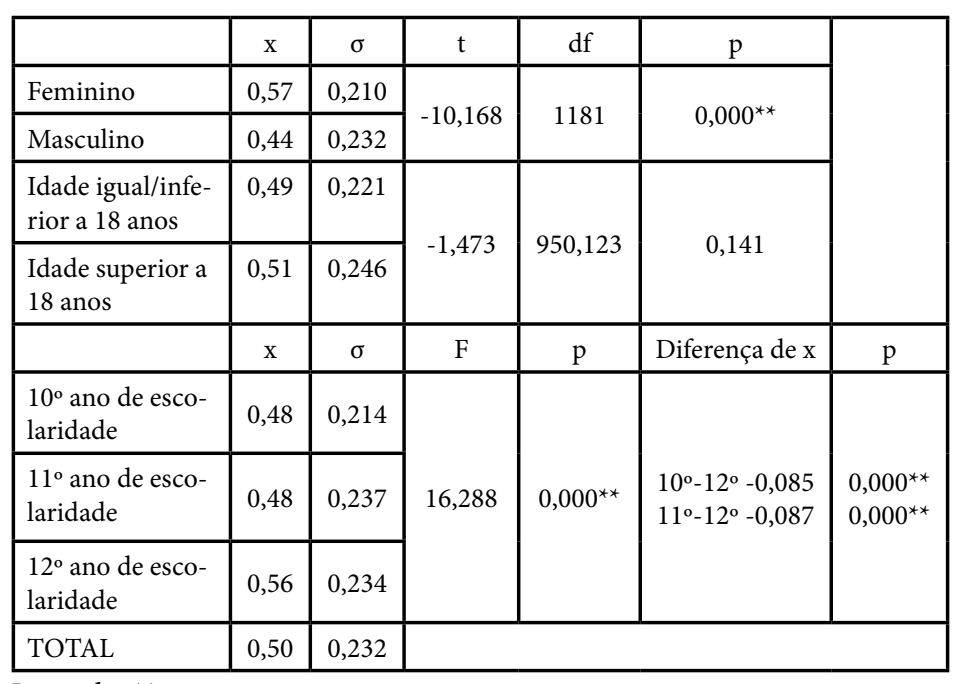

Legenda: ${ }^{* *}-\mathrm{p}<0,01$

Considerando as fontes de informação em saúde, as mais utilizadas pelos jovens inquiridos são médico $(\mathrm{x}=$ $3,68 ; \delta=1,077)$, seguido da família $(\mathrm{x}=3,67 ; \delta=0,851)$, da televisão $(\mathrm{x}=2,92 ; \delta=0,830)$, da internet $(\mathrm{x}=2,86$; $\delta=0,973)$, dos amigos $(\mathrm{x}=2,59 ; \delta=0,863)$, dos panfletos/brochuras $(\mathrm{x}=2,50 ; \delta=0,982)$, das revistas $(\mathrm{x}=2,41$; $\delta=0,952)$ e do farmacêutico $(x=2,29 ; \delta=1,099)$. As raparigas utilizam mais todas estas fontes de informação (Tabela 2). O médico é mais utilizado com fonte de informação pelos mais jovens e os amigos pelos mais velhos. OS jovens a frequentar anos de escolaridade superiores ( $12^{\circ}$ ano) utilizam mais as fontes de informação em saúde amigos e revistas. 
Tabela 2 - Utilização de fontes de informação em saúde (Teste t-student, One-Way ANOVA e Post-Hoc Tukey)

\begin{tabular}{|c|c|c|c|c|c|c|c|c|}
\hline Fonte de informação & & Feminino & Masculino & $10^{\circ}$ ano & $11^{\circ}$ ano & $12^{\circ}$ ano & Idade $<18$ anos & Idade $>18$ anos \\
\hline \multirow[t]{2}{*}{ Farmácia } & $\mathrm{X} ; \sigma$ & 2,$37 ; 1,061$ & 2,$23 ; 1,128$ & 2,$26 ; 1,114$ & 2,$31 ; 1,081$ & 2,$32 ; 1,106$ & 2,$27 ; 1,096$ & 2,$33 ; 1,103$ \\
\hline & df; $p$ & \multicolumn{2}{|c|}{$1199 ; 0,028^{*}$} & \multicolumn{3}{|c|}{$1200 ; 0,702$} & \multicolumn{2}{|c|}{$1199 ; 0,321$} \\
\hline \multirow[t]{2}{*}{ Televisão } & $\mathrm{X} ; \sigma$ & 3,$00 ; 0,816$ & 2,$84 ; 0,834$ & 2,$88 ; 0,855$ & 2,$91 ; 0,798$ & 2,$97 ; 0,839$ & 2,$92 ; 0,824$ & 2,$92 ; 0,837$ \\
\hline & $\mathrm{df} ; \mathrm{p}$ & \multicolumn{2}{|c|}{1203,$377 ; 0,001^{\star *}$} & \multicolumn{3}{|c|}{$1214 ; 0,283$} & \multicolumn{2}{|c|}{$1213 ; 0,959$} \\
\hline \multirow[t]{2}{*}{ Revistas } & $\mathrm{X} ; \sigma$ & 2,$64 ; 0,914$ & 2,$20 ; 0,938$ & 2,$32 ; 0,990$ & 2,$45 ; 0,894$ & 2,$47 ; 0,977$ & 2,$39 ; 0,950$ & 2,$45 ; 0,955$ \\
\hline & df; $p$ & \multicolumn{2}{|c|}{$1211 ; 0,000^{\star *}$} & \multicolumn{3}{|c|}{$1212 ; 0,050^{*}$} & \multicolumn{2}{|c|}{$1211 ; 0,301$} \\
\hline \multirow[t]{2}{*}{ Médico } & $\mathrm{X} ; \sigma$ & 3,$77 ; 1,034$ & 3,$59 ; 1,108$ & 3,$72 ; 1,072$ & 3,$66 ; 1,034$ & 3,$64 ; 1,149$ & 3,$74 ; 1,033$ & 3,$58 ; 1,135$ \\
\hline & df; $p$ & \multicolumn{2}{|c|}{1206,$554 ; 0,003^{\star *}$} & \multicolumn{3}{|c|}{$1210 ; 0,580$} & \multicolumn{2}{|c|}{980,$568 ; 0,018^{\star}$} \\
\hline \multirow[t]{2}{*}{ Internet } & $\mathrm{X} ; \sigma$ & 3,$00 ; 0,919$ & 2,$74 ; 1,005$ & 2,$78 ; 1,061$ & 2,$90 ; 0,908$ & 2,$92 ; 0,951$ & 2,$83 ; 0,984$ & 2,$91 ; 0,958$ \\
\hline & df; $p$ & \multicolumn{2}{|c|}{1208,$622 ; 0,000^{* *}$} & \multicolumn{3}{|c|}{$1210 ; 0,113$} & \multicolumn{2}{|c|}{$1209 ; 0,164$} \\
\hline \multirow[t]{2}{*}{ Família } & $\mathrm{X} ; \sigma$ & 3,$74 ; 0,836$ & 3,$62 ; 0,860$ & 3,$69 ; 0,895$ & 3,$65 ; 0,826$ & 3,$69 ; 0,828$ & 3,$69 ; 0,856$ & 3,$65 ; 0,843$ \\
\hline & df; $p$ & \multicolumn{2}{|c|}{$1213 ; 0,013^{*}$} & \multicolumn{3}{|c|}{$1214 ; 0,750$} & \multicolumn{2}{|c|}{$1213 ; 0,383$} \\
\hline \multirow[t]{2}{*}{ Amigos } & $\mathrm{X} ; \sigma$ & 2,$70 ; 0,819$ & 2,$49 ; 0,889$ & 2,$48 ; 0,878$ & 2,$61 ; 0,823$ & 2,$70 ; 0,885$ & 2,$52 ; 0,846$ & 2,$68 ; 0,880$ \\
\hline & df; p & \multicolumn{2}{|c|}{1208,$630 ; 0,000^{\star *}$} & \multicolumn{3}{|c|}{$1211 ; 0,002^{\star *}$} & \multicolumn{2}{|c|}{$1210 ; 0,002^{\star *}$} \\
\hline \multirow[t]{2}{*}{ Panfletos } & $\mathrm{X} ; \sigma$ & 2,$72 ; 0,963$ & 2,$30 ; 0,958$ & 2,$55 ; 1,054$ & 2,$48 ; 0,939$ & 2,$46 ; 0,947$ & 2,$53 ; 1,008$ & 2,$45 ; 0,942$ \\
\hline & df; $p$ & \multicolumn{2}{|c|}{$1210 ; 0,000^{\star *}$} & \multicolumn{3}{|c|}{$1211 ; 0,401$} & \multicolumn{2}{|c|}{$1210 ; 0,135$} \\
\hline
\end{tabular}

Legenda: ${ }^{\star}-\mathrm{p}<0,05 ;{ }^{* *}-\mathrm{p}<0,01$

Em termos correlacionais, a utilização de determinadas fontes de informação em saúde correlacionam-se de forma positiva com os níveis de literacia em saúde sobre uso de substâncias, nomeadamente a utilização de revistas $(\mathrm{r}=0,067 ; \mathrm{p}=0,021)$, do médico $(\mathrm{r}=0,079$; $\mathrm{p}=0,006)$, da internet $(\mathrm{r}=0,089 ; \mathrm{p}=0,002)$, dos amigos $(\mathrm{r}=0,059 ; \mathrm{p}=0,042)$ e dos panfletos/brochuras $(\mathrm{r}=0,084$; $\mathrm{p}=0,004)$.

Uma percentagem da variância da literacia em saúde sobre uso de substâncias explica-se pela utilização das fontes de informação em saúde (Tabela 3), na amostra total ( $\mathrm{r} 2=0,026 ; \mathrm{p}=0,000)$, e em todos os grupos da amostra excetuando as raparigas onde não foram encontradas predições.

Tabela 3 - Impacto do uso das fontes de informação em saúde na literacia em saúde sobre o uso de substâncias

(Teste Regressão Linear, método Enter)

\begin{tabular}{|l|l|l|l|l|}
\hline & $\mathrm{r}$ & $\mathrm{r}^{2}$ & $\mathrm{~F}$ & $\mathrm{p}$ \\
\hline Feminino & 0,090 & 0,008 & 0,552 & 0,817 \\
\hline Masculino & 0,184 & 0,034 & 2,644 & $0,007^{* *}$ \\
\hline Idade igual/inferior a 18 anos & 0,176 & 0,031 & 2,730 & $0,006^{* *}$ \\
\hline Idade superior a 18 anos & 0,189 & 0,036 & 2,119 & $0,033^{*}$ \\
\hline $10^{\circ}$ ano de escolaridade & 0,217 & 0,047 & 2,402 & $0,015^{\star}$ \\
\hline $11^{\circ}$ ano de escolaridade & 0,145 & 0,021 & 1,197 & 0,299 \\
\hline $12^{\circ}$ ano de escolaridade & 0,240 & 0,058 & 2,267 & $0,023^{*}$ \\
\hline TOTAL & 0,162 & 0,026 & 3,861 & $0,000^{* *}$ \\
\hline
\end{tabular}

Legenda: ${ }^{\star}-\mathrm{p}<0,05 ;{ }^{* *}-\mathrm{p}<0,01$
$\mathrm{Na}$ amostra total, contribuíram para a predição de $15,9 \%$ da variância da literacia em saúde sobre uso de substâncias, a utilização das fontes de informação panfletos/brochuras, farmacêutico, médico e internet, tal como se observa na Tabela 4 .

Tabela 4 - Impacto das fontes de informação em saúde na literacia em saúde sobre uso de substâncias (método Stepwise)

\begin{tabular}{|l|l|l|l|}
\hline & $\mathrm{r}$ & $\mathrm{r}^{2}$ & $\mathrm{p}$ \\
\hline Panfletos/brochuras & 0,093 & 0,009 & $0,001^{\star *}$ \\
\hline Panfletos/brochuras + Farmacêutico & 0,118 & 0,014 & $0,000^{\star *}$ \\
\hline $\begin{array}{l}\text { Panfletos/brochuras + Farmacêutico + } \\
\text { Médico }\end{array}$ & 0,147 & 0,022 & $0,000^{\star *}$ \\
\hline $\begin{array}{l}\text { Panfletos/brochuras + Farmacêutico + } \\
\text { Médico + Internet }\end{array}$ & 0,159 & 0,025 & $0,000^{\star *}$ \\
\hline
\end{tabular}

Legenda: ${ }^{*} \mathrm{p}<0,05 ;{ }^{*} \mathrm{p}<0,01$

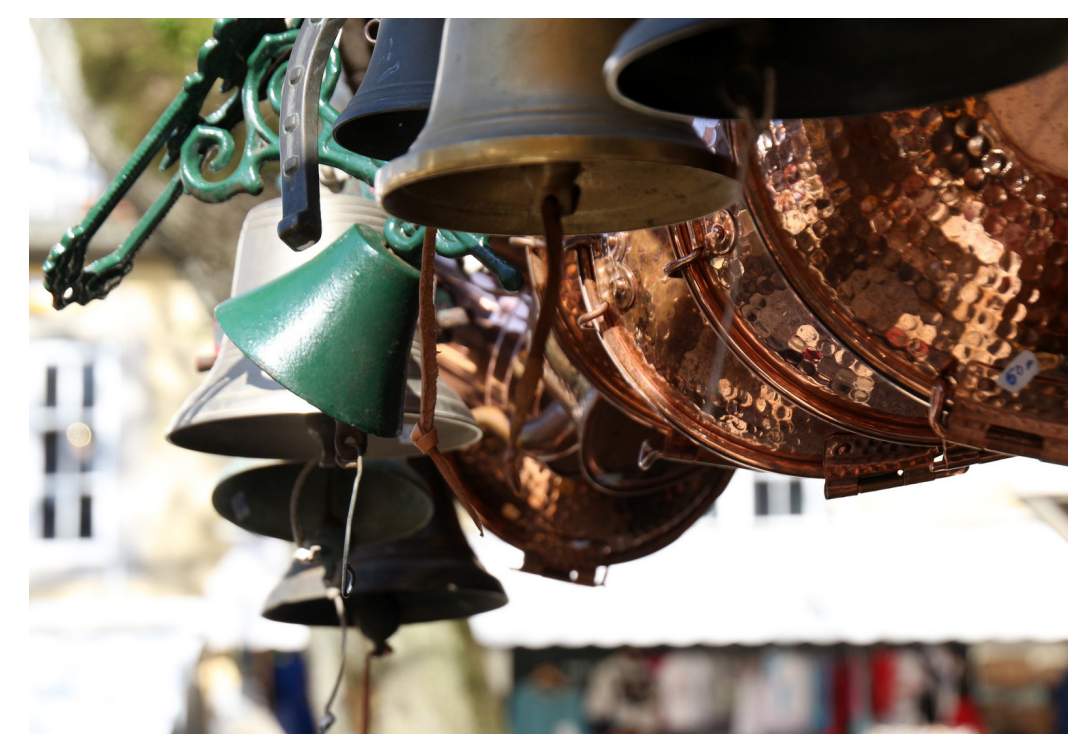




\section{DISCUSSÃO}

Foram encontrados níveis de literacia sobre uso de substâncias satisfatórios, apesar de não serem muito elevados, o que confirma a informação referida na literatura. Grande parte dos autores, tal como Sanders et al. (2009), referem que os níveis de literacia em saúde na população geral e na população adolescentes em particular são baixos, demonstrando poucas competências para encontrar, avaliar, compreender, e utilizar a informação sobre saúde. Tal como (Wu, et al., 2010) também os nossos resultados permitem perceber que as raparigas apresentam níveis de literacia em saúde sobre uso de substâncias superiores aos rapazes, tendo sido também percebido que estes níveis são superiores em jovens a frequentar o $12^{\circ}$ ano de escolaridade e não variam com a idade dos mesmos.

Poderiam ser esperados níveis superiores de literacia em saúde nesta temática, dado que os jovens referem ser um tema sobre o qual sentem necessidade e interesse em encontrar informação (Gray et al., 2005), sendo um dos tópicos privilegiados em intervenção nesta faixa etária (Ackard \& Neumark-Sztainer, 2001, AlQallaf, Al-Otaibi, \& Othman, 2012, Kwan et al., 2010). Também os rapazes afirmam, de acordo com os autores acima citados, receber mais informação sobre uso de substâncias, pelo que se esperaria encontrar valores mais elevados neste grupo. Denota-se então que apesar de receberem mais informação sobre o assunto, os rapazes poderão ter menos competências de literacia em saúde para utilizar esta informação.

As fontes de informação mais utilizadas pelos jovens são o médico e família, fontes estas que com a idade, vão sendo substituídas pelos amigos, sendo que as raparigas utilizam mais todas estas fontes de informação. Os jovens a frequentar anos de escolaridade superior utilizam mais as fontes de informação revistas e amigos.

Estes resultados, semelhantes aos encontrados na literatura (Dobransky \& Hargittai, 2012, Kwan et al., 2010, Nustad, Adams, \& Moore, 2008), demonstram uma valorização dos jovens em relação às fontes de informação em saúde fidedignas, como os pais e os profissionais de saúde, em detrimentos dos amigos, sendo as raparigas que procuram mais informação sobre saúde. No entanto, as fontes de informação como o médico vão, à medida que os adolescentes se tornam mais velhos, sendo substituídas pelos amigos e pelas revistas, fontes de informação menos credíveis, mas que exercem uma pressão social mais elevada.
Com a aquisição de autonomia dos jovens, que decorre com a idade, a necessidade de responder a expectativas sociais e de grupo, bem como a necessidade de identificação com os pares leva a que esta fonte de informação passe a ser privilegiada, mesmo com a valorização científica dos profissionais de saúde.

Uma percentagem da variância da literacia em saúde sobre uso de substâncias explica-se pela utilização das fontes de informação em saúde, na amostra total, bem como na maioria dos grupos da amostra, contribuindo para esta predição a utilização das fontes de informação Panfletos/brochuras, Farmacêutico, Médico e Internet. Tal como referem Kutner, Greenberg, Jin, Paulsen, \& White (2006) e Paek, Reber, \& Lariscy (2011), a utilização mais frequente de fontes de informação em saúde e a pesquisa de informação sobre saúde leva a um aumento dos níveis de literacia em saúde, o que suporta os resultados encontrados, em relação à literacia em saúde sobre uso de substâncias.

\section{CONCLUSÃO}

A amostra apresenta níveis razoáveis de literacia em saúde sobre uso de substâncias, e uma frequente consulta de fontes de informação em saúde, sendo as fontes mais utilizadas o médico e a família. No entanto, com a idade, os participantes passam a procurar mais informação sobre saúde junto dos amigos. Os valores mais elevados de literacia em saúde sobre uso de substâncias encontram-se em adolescentes do sexo feminino e a frequentar o $12^{\circ}$ ano de escolaridade, não havendo relação estatisticamente significativa destes níveis com a idade. Foi percebida uma relação de causalidade estatisticamente significativa, apesar de fraca entre os níveis de literacia em saúde sobre uso de substâncias e a utilização das fontes de informação em saúde, nomeadamente o Panfletos/brochuras, Farmacêutico, Médico e Internet no grupo estudado.

\section{IMPLICAÇÕES PARA A PRÁTICA CLÍNICA}

Analisando estes resultados denota-se a necessidade continuar a estudar este conceitos na população adolescente. A compreensão das competências de literacia em saúde nesta população permitirão compreender o impacto das intervenções realizadas com o objetivo de a promover. Incluir fontes de informação em saúde valorizadas pelos jovens (profissionais de saúde como enfermeiros e educação pelos pares) 'poderá influenciar os resultados positivos em termos de competências de literacia, tal como se perceber pelos resultados obtidos. 


\section{REFERÊNCIAS BIBLIOGRÁFICAS}

Ackard, D. M. \& Neumark-Sztainer, D. (2001). Health Care Information Sources for Adolescents: Age and Gender Differences on Use, Concerns, and Needs. Journal of Adolescent Health, 29(3), 170-176. doi: 10.1016/ S1054-139X(01)00253-1

Al-Qallaf, B. A., Al-Otaibi, B. N., \& Othman, H. A. (2012). Perception of Adolescents Towards health Related Issues in Secondary Schools, Kuwait. European Journal of General Medicine, 9(3), 162-169.

Andreassen, H. K., Bujnowska-Fedak, M. M., Chronaki, C. E., Dumitri, R. C., Pudule, I., Santana, S., Voss, H., Wynn, R. (2007). European citizens' use of Ehealth services: A study of seven countries. BMC Public Health, 7, 53. doi: 10.1186/1471-2458-7-53

Austin, R. (2012). eHealth literacy for older adults Part I. ANIA-CARING Newsletter, 1, 7-9.

Dobransky, K. \& Hargittai, E. (2012). Inquiring Minds Acquiring Wellness: Uses of Online and Offline Sources of Health Information. Health Communication, 27(4), 331-343. doi: 10.1080/10410236.2011.585451

Ghaddar, S. F., Valerio, M. A., Garcia, C. M., \& Hansen, L. (2012). Adolescent Health Literacy: The Importance of Credible Sources for Online Health Information. Journal of School Health, 82(1), 28-36. doi: 10.1111/j.1746-1561.2011.00664.x

Gray, N. J., Klein, J. D., Noyce, P. R., Sesselberg, T. S., \& Cantrill, J. A. (2005). Health information-seeking behaviour in adolescence: the place of the internet. Social Science \& Medicine, 60(7), 1467-1478. doi:10.1016/j. socscimed.2004.08.010

Kutner, M., Greenberg, E., Jin, Y., Paulsen, C., \& White, S. (2006). The Health Literacy of America's Adults: Results From the 2003 National Assessment of Adult Literacy (NCES 2006-483). U.S. Department of Education. Washington, DC, United States of America: National Center for Education.

Kwan, M. Y., Arbour-Nicitopoulos, K. P., Lowe, D., Taman, S., \& Faulkner, G. E. (2010). Student Reception, Sources, and Believability of Health-Related Information. Journal of American College Health, 58(6), 555562. doi: 10.1080/07448481003705925
Mukherjee, A., \& Bawden, D. (2012). Health information seeking in the information society. Health Information and Libraries Journal, 29(3), 242-246. doi: 10.1111/j.1471-1842.2012.00997.x

Norman, C. D., \& Skinner, H. A. (2006). eHEALS: The eHealth Literacy Scale. Journal of Medical Internet Research, 8(4), e27. doi: 10.2196/jmir.8.4.e27

Nustad, J., Adams, T., \& Moore, M. (2008). Health Information Sources Accessed by College Females: Differences Between Body-Image Distorted and NonBody-Image Distorted. Health Marketing Quarterly, 25(3), 241-253. doi: 10.1080/07359680802081837

Paek, H.-J., Reber, B. H., \& Lariscy, R. W. (2011). Roles of interpersonal and media socialization agents in adolescent self-reported health literacy: a health socialization perspective. Health Education Research, 26(1), 131-149. doi: 10.1093/her/cyq082

Sanders, L. M., Shaw, J. S., Guez, G., Baur, C., \& Rudd, R. (2009). Health Literacy and Child Health Promotion: Implications for Research, Clinical Care, and Public Policy. Pediatrics 124(3), S306-S314. doi: 10.1542/ peds.2009-1162G

Stellefson, M., Hanik, B., Chaney, B., Chaney, D., Tennant, B., \& Chavarria, E. A. (2011). eHealth Literacy Among College Students: A Systematic Review With Implications for eHealth Education. Journal of Medical Internet Research, 13(4), e102. doi: 10.2196/jmir.1703

Tomás, C. (2014). Literacia em saúde na adolescência. Dissertação para obtenção do Grau de Doutor em Ciências de Enfermagem, Universidade do Porto (Instituto de Ciências Biomédicas Abel Salazar), Portugal.

Vardavas, C. I., Kondilis, B. K., Patelarou, E., Akrivos, P. D., \& Falagas, M. E. (2009). Health literacy and sources of health education among adolescents in Greece. International journal of adolescent medicine and health, 21(2), 179-186.

Wu, A. D., Begoray, D. L., MacDonald, M., Higgins, J. W., Frankish, J., Kwan, B., Fung, W., Rootman, I. (2010). Developing and evaluating a relevant and feasible instrument for measuring health literacy of Canadian high school students. Health Promotion International, 25(4), 444-452. doi: 10.1093/heapro/daq032 